\section{Inauguration of CERN SPS}

\section{May 7}

Twenty five years to the day after the first meeting of a new inter-governmental group that quickly became known by its acronym CERN, the latest of the Organization-'s high energy ex perimental tools, the $400 \mathrm{GeV}$ SPS was formally inaugurated. At the ceremony, presided over by P. Levaux, President of Council, and attended by representatives of governments, local authorities, the world's high energy physics community, sister organizations and CERN staff, the notion of collaborative endeavour was emphasized by the absence of a single person to press a symbolic button or open an imaginary door. Just the flags were flown in tribute to the 12 member states of CERN.

In his talk reviewing the history of the project, the 2000-strong audience was reminded by $\mathrm{E}$. Amaldi, first Secretary-General of CERN and President of the Council when the decision to build the SPS was taken, that the first plans for the SPS were already being laid in 1960. W. Paul, Chairman of the Scientific Policy Committee, recalled the unique size of the machine and the technological innovations that had gone into its realization. It was a source of gratification to scientists and administrators alike that such a large enterprise had been completed ahead of time and with money to spare. This was continuing one of the traditions of the Organization that member states much appreciated.

But hand in hand with the construction of the machine had been the preparation of the experimental programme, starting immediately after the decision with meetings of physicists from all over Europe. The result of this early consultation was that 43 experiments had already been approved, in volving some 900 experimenters from over 100 laboratories. It was appropriate then that C. Llewellyn-Smith, as representing the younger generation of physicists who would be profiting from these years of preparation, should pass in review the exciting discoveries of recent times and evoke the tantalising prospect of a unification of the four forces (following the identification of neutral currents, charm and the fourth quark) and the possibility of combining the properties

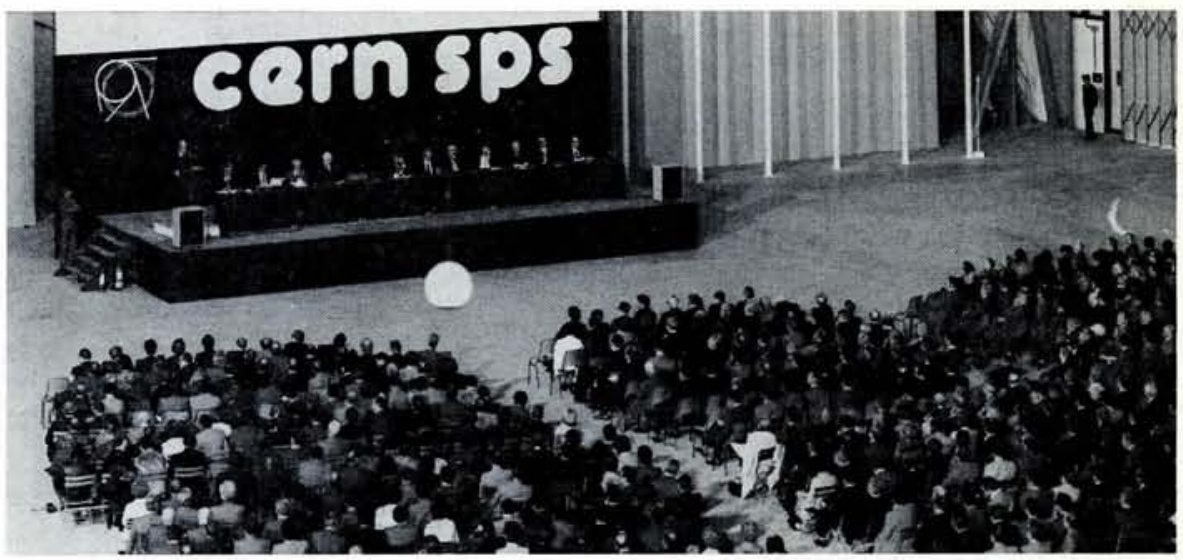

The hadron experimental hall prepared for the occasion. On the rostrum are left to right: W. Paul (speaking), J.B. Adams, Ch. Llewellyn-Smith, E. Amaldi, N. Ramsey, P. Levaux, B. P. Gregory, A. A. Logunov, V. F. Weisskopt, W. Jentschke, A. C. Pappas and L. Van Hove.

of hadrons and leptons into a single family.

The ceremony ended with a film ("Matter of Substance") introduced by J.B. Adams, executive director-general of CERN, which stood back from the nuts and the bolts, the magnets and the computers and tried to put into perspective this man-made device in a man-made environment. In so doing, it was echoing the thoughts of P. Graber, Swiss Minister for Foreign Affairs, who at a preparatory lunch given by the Host States across whose territory CERN is built, introduced the theme that man is no longer affronting just an abstract Nature, but also the situations he has created and, therefore, himself. Research must not be just an end in itself but rather a means of promoting the future well-being of humanity.

\section{Reference}

1. GOLDSMITH, M. and SHAW, E.N. Europe's Giant Accelerator (Taylor and Francis, London) 1977.

\title{
Magnetic Field of a Neutron Star Measured with Precision
}

For the first time the magnetic field of a neutron star has been measured with precision and in the process the strongest magnetic field so far to be detected has been revealed. It has the impressive value of $4.6 \times 10^{8}$ Tesla. The measurement was carried out by a collaboration of the University of Tubingen and the Max-Planck-Institute for Extraterrestrial Physics. From a $100 \mathrm{~m}$ diameter balloon, flown at an altitude of $42 \mathrm{~km}$, the X-ray emissions from the double-star Hercules X-1 were studied using solid-state detectors able to record up to an energy of $80 \mathrm{keV}$. This led to the identification of a $53 \mathrm{keV}$ cyclotron radiation line.

Hercules $\mathrm{X}-1$ is a double star consisting of a normal star, estimated to be $2 \mathrm{Gm}$ diameter, round which on an orbit of radius $3 \mathrm{Gm}$ rotates a 20 $\mathrm{km}$ diameter neutron star. Matter emitted from the main star is drawn towards the neutron star and while some is trapped by the star's magnetic field in a plasma ring some $100 \mathrm{Mm}$ wide, some is able to spiral down towards the magnetic poles creating a hot spot there (raising the temperature to the order of $100 \mathrm{MK}$ ) and giving rise to the cyclotron emission line.
Identification comes from the characteristic frequency of the X-ray pulsations. The orbiting period of the neutron star is 1.7 day and the period of rotation on its own axis : $1.24 \mathrm{~s}$. As the axes of rotation and magnetic field are inclined relative to each other, the emission area disappears below the horizon every $1.24 \mathrm{~s}$. Moreover, as the neutron star is eclipsed behind the principal star every 1.7 day, there is a further regular fluctuation from this cause. What is not clear is why there is a 12-day and 23-day periodicity also in the star's emission.

Professor R. Kippenhahn, Director of the Max-Planck-Institute describes the measurements as "probably the most important observations made by German astronomers since the War... For the first time the strength of the magnetic field of a neutron star has been measured with an accuracy of about $10 \%$ and as a result, we now have a key to understanding the bizarre physics of the surface of neutron stars." In September, the experiment will be repeated with bigger and more refined $\mathrm{X}$-ray detectors and no doubt other observatories will be studying this remarkable object. 